essierte Allergologen und allergologisch interessierte Immunologen ein Forum sein für einen fruchtbaren gegenseitigen Austausch. Sie steht allen interessierten Mitgliedern der DGAI offen. Eine Kontaktaufnahme ist am besten über den jeweiligen Sprecher der Sektion möglich, derzeit: Prof. Dr. Joachim Saloga, Universitäts-Hautklinik Mainz; Stellvertreter: Prof. Dr. Albrecht Bufe, Experimentelle Pneumologie, Berg-

\section{DGAI-Sektionstreffen}

Die Sektionen der Deutschen Gesellschaft für Allergologie und klinische Immunologie (DGAI) treffen sich zum nächsten Mal auf der 22. DGAl-Tagung in Bochum, am 14. März 2002, 7:45-8:45 Uhr (s. Kongressprogramm für die jeweiligen Orte). Die Sitzung der Sektion Pneumologie/Innere Medizin findet um 12:00-13:00 Uhr statt.

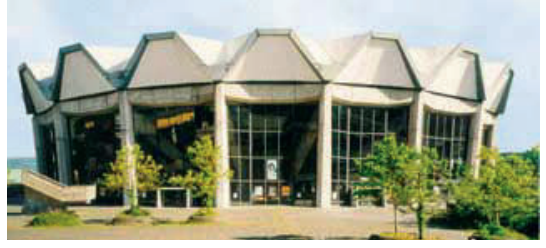

\section{Sektion Pädiatrie}

D ie DGAI-Sektion Pädiatrie bereitet derzeit ein gemeinsames Positionspapier mit der GPA (Gesellschaft für Pädiatrische Allergologie und Umweltmedizin) zum Thema „Alimentäre Atopie-Prävention “ vor. Hier sollen vor allem auch die neuesten Ergebnisse der GINI-Daten mit berücksichtigt werden. In dieses Positionspapier ist auch Frau Dr. Schmid von der DISA eingebunden.

\section{Prof. Dr. Carl Peter Bauer}

Sprecher der Sektion Pädiatrie

Fachklinik Gaißach

Klinik für chronische Erkrankungen im

Kindes- und Jugendalter

83674 Gaißach bei Bad Tölz

Telefon: o 8041 / 798221

Telefax: o $8041 / 798222$

E-Mail:info@fachklinik-gaissach.de mannsheil, Univ.-Klinik, Bochum. Um die Angabe der eigenen E-Mail-Adresse für die weitere Kommunikation wird dringend gebeten.

\section{Prof. Dr. Joachim Saloga}

Sprecher der Sektion Immunologie

Universitäts-Hautklinik Mainz

Langenbeckstr. 1, 55131 Mainz

Telefax: 06131-17473751

E-Mail:saloga@hautklinik.klinik.unimainz.de
Die DGAI-Geschäftsstelle

hat ein neues Postfach

Deutsche Gesellschaft für Allergologie und klinische Immunologie DGAI Erika Ratzinger

Postfach 700464

81304 München

Nach wie vor gültig sind:

Tel.: 089 / $5466-2968$

Fax: $089 / 5838-24$

E-mail: RatzingerErika@t-online.de

\title{
Sektion Innere Medizin und Pneumologie
}

D ie Fertigstellung und Erprobung des „Modul 1 Allergologie“ im Rahmen eines aus drei Modulen bestehenden Curriculums für die Ausbildung zur pneumologischen Fachassistentin stand $2001 \mathrm{im}$ Mittelpunkt der DGAI-Sektion Innere Medizin und Pneumologie.

Das Gesamtmaterial umfasst neben dem „Modul 1 Allergologie“ das Modul 2 mit der Funktionsdiagnostik und fasst im Modul 3 die Gebiete Patientenschulung, Schlafapnoe, Bronchiologie und andere Gebiete zusammen.

Das „Modul 1 Allergologie“ vermittelt auf 106 Seiten folgende wesentliche Inhalte:

- Die Definition der Allergie und ihre wesentlichen pathophysiologischen Grundlagen.

— Der Abschnitt „Mit dem Allergen

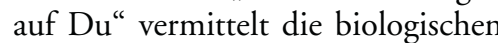
Charakteristika saisonaler und perennialer Allergene, ihr Auftreten und ihre Besonderheiten.

— „Allergologisches Handwerkszeug 1“ vermittelt die Indikationen, Durchführung und Beurteilung der Hauttests, einen Vergleich der verschiedenen Arten von Hauttests, ihre Dokumentation und Auswertung.

—Zudem vermittelt „Allergologisches Handwerkszeug 1" Kenntnisse in den organbezogenen Provokationstests an der Nase und an den Bronchien. Daneben werden die In-vitroTests besprochen.

—Der Abschnitt „Nahrungsmittel“ vermittelt Kenntnisse zur Differen- zierung von Allergie und Intoleranz sowie klinische Aspekte der Nahrungsmittel-Allergie.

— „Antiallergische Therapie 1“ gibt einen Überblick über die Medikamente zur Behandlung allergischer Erkrankungen und detaillierte Hinweise zur Allergen-Vermeidung.

— Die „Antiallergische Therapie 2“ ist der spezifischen Immuntherapie gewidmet.

Das gesamte Material ist didaktisch sehr geschickt aufgebaut. Es wurde durch mehrere Mitglieder der Arbeitsgruppe inhaltlich geprüft und in der praktischen Anwendung als außerordentlich sinnvoll eingeschätzt. Die Federführung und die meiste Arbeit an dem Material ist von Herrn Dr. med. Rolf Kroidl in Stade geleistet worden, wofür ihm die Arbeitsgruppe sehr dankt. Das Curriculum wurde auch von anderen Fachdisziplinen akzeptiert und zum Teil eingesetzt.

Die Arbeitsgruppe hat die Planung für ein Themenheft der Zeitschrift Allergo Journal unter dem Titel „Allergen-Meidung und Prävention allergischer Atemwegserkrankungen" abgeschlossen, und sie wird sich im Jahre 2002 auf die Erstellung der Manuskripte konzentrieren.

Prof. Dr. Karl-Christian Bergmann Sprecher der Sektion Innere Medizin und Pneumologie

Allergie- und Asthma-Klinik Wilhelm Gronemeyer, An der Martiniusquelle 10 33175 Bad Lippspringe

Telefon: 05252 / 952402

Telefax: 05252 / 952403

E-Mail: aak-bergmann@t-online.de 